\title{
The Temperature and Pressure Dependence of the Free Volume in Fluoroelastomers from PALS and PVT Experiments
}

G. Dlubek ${ }^{a}$, A. Sen Gupta ${ }^{b, c}$ J. WawryszczuK ${ }^{d}$, D. Kilburn ${ }^{e}$, J. Pionteck ${ }^{f}$, R. Krause-RehberG ${ }^{b}$, T. GoworeK $^{d}$,

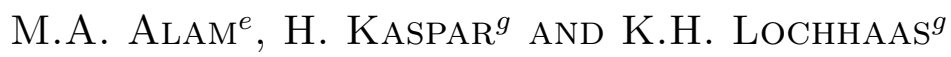

${ }^{a}$ ITA Inst. Innovative Technologies

Wiesenring 4, 06120 Lieskau (Halle/S.), Germany

${ }^{b}$ Department of Physics, Martin-Luther-University, 06099 Halle/S., Germany

${ }^{c}$ Dep. of Physics, Visva-Bharati, Central University

Santiniketan, West Bengal-731235, India

${ }^{d}$ Institute of Physics, Maria Curie-Skłodowska University, 20-031 Lublin, Poland

${ }^{e}$ H.H. Wills Physics Laboratories, Univ. of Bristol

Tyndall Avenue, Bristol, BS8 1TL, UK

${ }^{f}$ Leibniz-Institute of Polymer Research, Hohe Strasse 6, 01069 Dresden, Germany ${ }^{g}$ Dyneon GmbH \& Co. KG, Werk Gendorf, 84504 Burgkirchen, Germany

Positron lifetime and pressure-volume-temperature experiments were performed as function of $T(100-485 \mathrm{~K})$ and $P\left(10^{-5} \mathrm{~Pa}-500 \mathrm{MPa}\right)$ on a fluoroelastomer. From positron annihilation lifetime spectroscopy, analyzed with LT9.0 assuming a dispersion in the $\mathrm{e}^{+}\left(\tau_{2}\right)$ and $o$-Ps $\left(\tau_{3}\right)$ lifetime, the mean, $\left\langle v_{\mathrm{h}}\right\rangle$, and the width (standard deviation), $\sigma_{\mathrm{h}}$, of the hole size distribution were calculated. The pressure-volume-temperature data were analyzed using the Simha-Somcynsky hole-lattice theory to estimate the specific hole free volume $V_{\mathrm{f}}$. From a comparison of $V_{\mathrm{f}}$ with $\left\langle v_{\mathrm{h}}\right\rangle$ the specific hole number, $N_{\mathrm{h}}^{\prime}$, is estimated. The data from thermal expansion and pressure experiments show complete agreement when taking into account the compressibility of the occupied volume.

PACS numbers: 61.41.+e, 78.70.--g

\section{Introduction}

From positron annihilation lifetime spectroscopy (PALS) experiments the free volume hole size distribution, its mean and width, but not directly the num- 
ber density and the hole free volume fraction, can be estimated. The latter value, however, can be obtained from the analysis of pressure-volume-temperature experiments using the Simha-Somcynsky theory ([1] and [2-4]). This theory models the disordered structure of a liquid by a cell or lattice model with lattice sites randomly occupied by a mer with a fraction $y$ of less than one. From a fit of the equation of state (S-S eos) to the specific volume $V$ the occupied, $y$, and the unoccupied, $h=1-y$, volume fractions and from this specific occupied, $V_{\text {occ }}=y V$, and hole free, $V_{\mathrm{f}}=h V$, volumes can be calculated. In this work we demonstrate the potential of the comparison of PVT and PALS data in thermal expansion and hydrostatic compression experiments, and discuss briefly the question how to analyze correctly PALS data.

\section{Results and discussion}

The polymer under investigation is a commercial copolymer of tetrafluoroethylene and perfluoro(methyl vinyl ether) made by Dyneon GmbH \& Co. KG, Germany (a 3M Company) and known as perfluoroelastomer PFE. The PVT experiments were performed in the $T, P$ range of $T=295.8-485.3 \mathrm{~K}$ and $P=$ $10-200 \mathrm{MPa}$. Positron lifetime spectra $\left(2-6 \times 10^{6}\right.$ counts, resolution $\left.\approx 230 \mathrm{ps}\right)$ were measured in the range $T=100-473 \mathrm{~K}$ and $P=10^{-5} \mathrm{~Pa}$ (thermal expansion [3]) and $T=295.8 \mathrm{~K}$ and $P=0.1-448 \mathrm{MPa}$ (hydrostatic compression [4]). More conditions of the experiments can be found in Refs. [3, 4]. Figure 1 shows the results of the $\mathrm{S}-\mathrm{S}$ eos analysis of $\mathrm{PVT}$ experiments extrapolated to $P=0 \mathrm{MPa}$. The following coefficients of thermal expansion of the total, occupied, and free volumes at $300 \mathrm{~K}$ were estimated: $\alpha=8.67, \alpha_{\mathrm{occ}}=0.38$, and $\alpha_{\mathrm{f}}=72.4$ in units of $10^{-4} \mathrm{~K}^{-1}$. The corresponding values for the compressibility are

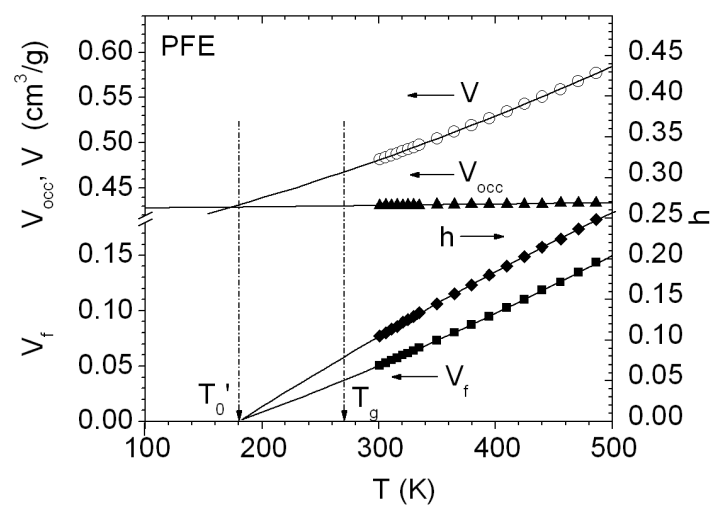

Fig. 1. The specific (total) volume $V$, the hole fraction $h$, and the specific occupied and free volumes $V_{\text {occ }}=(1-h) V$ and $V_{\mathrm{f}}=h V$ for PFE at $P=0$ Pa (extrapolated). The solid lines are linear $\left(V_{\text {occ }}\right)$ or quadratic fits $\left(V, V_{\mathrm{f}}, h\right)$ and extrapolations. $T_{\mathrm{g}}$ is from differential scanning calorimetry (DSC), and $T_{0}^{\prime}$ indicates the temperature where the extrapolated (hypothetical) hole free volume disappears. 
$\kappa=7.87, \kappa_{\text {occ }}=2.5$, and $\kappa_{\mathrm{f}}=53$ in units of $10^{-4} \mathrm{MPa}^{-1}$. The results show that $V_{\text {occ }}$ exhibits almost no thermal expansion (for $T>T_{\mathrm{g}}$ ) but is compressible. This result is in agreement with our previous study of polystyrene where we found the unexpected but important result: $\alpha_{\text {occ, } \mathrm{r}}\left(T>T_{\mathrm{g}}\right) \approx \alpha_{\mathrm{g}} / 10 \approx 0$, $\alpha_{\mathrm{occ}, \mathrm{g}}\left(T<T_{\mathrm{g}}\right) \approx \alpha_{\mathrm{g}} / 2\left(\alpha_{\mathrm{g}}=2.25 \times 10^{-4} \mathrm{~K}^{-1}\right)$ and $\kappa_{\mathrm{occ}, \mathrm{g}}\left(T<T_{\mathrm{g}}\right) \approx$ $\kappa_{\text {occ, } \mathrm{r}}\left(T>T_{\mathrm{g}}\right) \approx 2.2 \times 10^{-4} \mathrm{MPa}^{-1}[2]$.

The treatment of the positron lifetime spectra employing the routine LT9.0 [5] showed us that a correct analysis requires to allow both the $\mathrm{e}^{+}\left(\tau_{2}\right)$ and the $o$-Ps $\left(\tau_{3}\right)$ lifetime to be distributed (widths $\sigma_{2}$ and $\sigma_{3}$ ). In that case $p$-Ps parameters come out as theoretically expected: $\tau_{1} \approx 125 \mathrm{ps}(\eta \approx 1)$ and $I_{1} / I_{3}=1 / 3[3,4]$. From $\tau_{3}$ the mean hole radius can be calculated using a well known, semi-empirical equation, $r_{\mathrm{h}}=r_{\mathrm{h}}\left(\tau_{3}\right)$ and $v_{\mathrm{h}}=4 \pi r_{\mathrm{h}}^{3} / 3[6]$. Since the $o$-Ps lifetime has a broad distribution, it is more correct to calculate the mean hole volume as the mass centre of the hole size distribution. The radius probability distribution $n\left(r_{\mathrm{h}}\right)$ can be obtained from $n\left(r_{\mathrm{h}}\right)=-\alpha_{3}(\lambda) \mathrm{d} \lambda_{3} / \mathrm{d} r_{\mathrm{h}}$ where $\alpha_{3}(\lambda)$ is the $o$-Ps annihilation rate distribution [6] $\left(\alpha_{3}(\lambda) \times \lambda\right.$ is assumed to be a log normal function in LT9.0). The volume fraction hole size distribution follows from $g\left(v_{\mathrm{h}}\right)=n\left(r_{h}\right) / 4 \pi r_{\mathrm{h}}^{2}$ and the number fraction hole size distribution from $g_{\mathrm{n}}\left(v_{\mathrm{h}}\right)=g\left(v_{\mathrm{h}}\right) / v_{\mathrm{h}}$. Moreover, we calculated the modified distribution $g_{\mathrm{n}}\left(v_{\mathrm{h}}\right)^{*}=g_{\mathrm{n}}\left(v_{\mathrm{h}}\right) / v_{\mathrm{h}}$. The mean and the variance of $g_{\mathrm{n}}\left(v_{\mathrm{h}}\right),\left\langle v_{\mathrm{h}}\right\rangle$ and $\sigma_{\mathrm{h}}^{2}$, and of $g_{\mathrm{n}}\left(v_{\mathrm{h}}\right)^{*},\left\langle v_{\mathrm{h}}\right\rangle^{*}$ and $\sigma_{\mathrm{h}}^{* 2}$, were calculated numerically as first and second moment of these distributions (for more details see Refs. $[3,4])$. Figure 2 shows that the slopes of the $v_{\mathrm{h}}$ vs. $T$ curve and the extrapolation to $v_{\mathrm{h}}=0, \mathrm{~T}_{0}^{\prime}$, depend on the definition of $v_{\mathrm{h}}=v_{\mathrm{h}}\left(\tau_{3}\right),\left\langle v_{\mathrm{h}}\right\rangle$, or $\left\langle v_{\mathrm{h}}\right\rangle^{*} \cdot v_{\mathrm{h}}$ shows a normal glass transition $\left(T_{\mathrm{g}}\right)$ behaviour and a levelling off at $T_{\mathrm{k}}$.

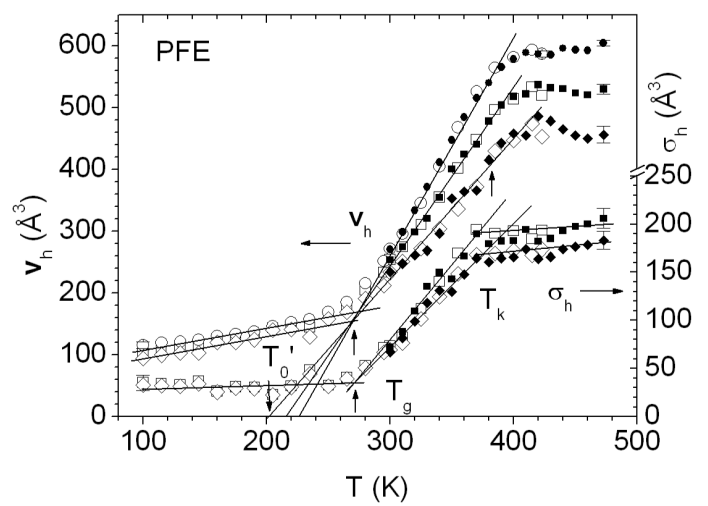

Fig. 2. Mean hole volume $v_{\mathrm{h}}$ for PFE calculated either as $v_{\mathrm{h}}=v_{\mathrm{h}}\left(\tau_{3}\right)$ (circles), the number average, $\left\langle v_{\mathrm{h}}\right\rangle$ (squares), or the corrected average, $\left\langle v_{\mathrm{h}}\right\rangle^{*}$ (diamonds), volume and the corresponding hole volume dispersions $\sigma_{\mathrm{h}}=\sigma_{\mathrm{h}}$ and $\sigma_{\mathrm{h}}^{*}$ at $P=10^{-5} \mathrm{~Pa}$. Empty and filled symbols: two PFE samples. $T_{\mathrm{k}}$ denotes the "knee" temperature, above $T_{\mathrm{k}}$ the $o$-Ps lifetime does not further mirror the true hole size. $T_{\mathrm{g}}$ and $T_{0}^{\prime}$ are given as in Fig. 1. The straight lines are due to linear fits. 


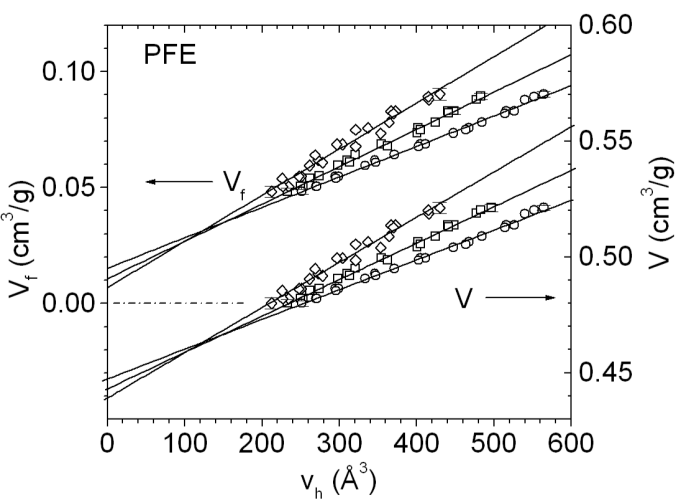

Fig. 3. Specific hole free volume $V_{\mathrm{f}}$ and specific total volume $V$ plotted vs. the mean hole volume calculated as $v_{\mathrm{h}}=v_{\mathrm{h}}\left(\tau_{3}\right)$ (circles), $\left\langle v_{\mathrm{h}}\right\rangle$ (squares), or $\left\langle v_{\mathrm{h}}\right\rangle^{*}$ (diamonds) for $\mathrm{PFE}$ (all for $P \approx 0 \mathrm{~Pa}$ ). The lines show linear fits to the data from the temperature range between $T_{\mathrm{g}}+30 \mathrm{~K}$ and $T_{\mathrm{k}}$.

In Fig. 3 plots of the specific hole free, $V_{\mathrm{f}}$, and total, $V$, volume vs. the $o$-Ps hole volume $v_{\mathrm{h}}$ are shown. The specific hole number $N_{\mathrm{h}}^{\prime}$ can be estimated from the relations

$$
V_{\mathrm{f}}=V_{\mathrm{f} 0}+N_{\mathrm{h}}^{\prime} v_{\mathrm{h}}
$$

or

$$
V=V_{\text {occ }}+V_{\mathrm{f} 0}+N_{\mathrm{h}}^{\prime} v_{\mathrm{h}} \text {. }
$$

The figure shows that $\left\langle v_{\mathrm{h}}\right\rangle^{*}$ corresponds best to the expectation $V_{\mathrm{f} 0}=0$. From this it follows that probably o-Ps detects holes with a preference which corresponds approximately to the hole volume $v_{\mathrm{h}}$. This preference is corrected in calculating $\left\langle v_{\mathrm{h}}\right\rangle^{*}$ as the mean of the modified distribution $g_{\mathrm{n}}\left(v_{\mathrm{h}}\right)^{*}=$ $g_{\mathrm{n}}\left(v_{\mathrm{h}}\right) / v_{\mathrm{h}}$. The specific hole number estimated from $N_{\mathrm{h}}^{\prime}=\mathrm{d} V_{\mathrm{f}} / \mathrm{d}\left\langle v_{\mathrm{h}}\right\rangle^{*}, N_{\mathrm{h}}^{\prime}=$

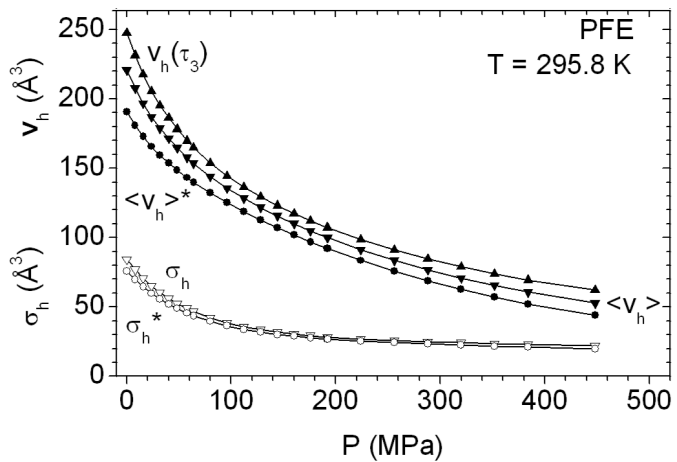

Fig. 4. As in Fig. 2, but as a function of the pressure $P$ at $T=295.8 \mathrm{~K}$. 


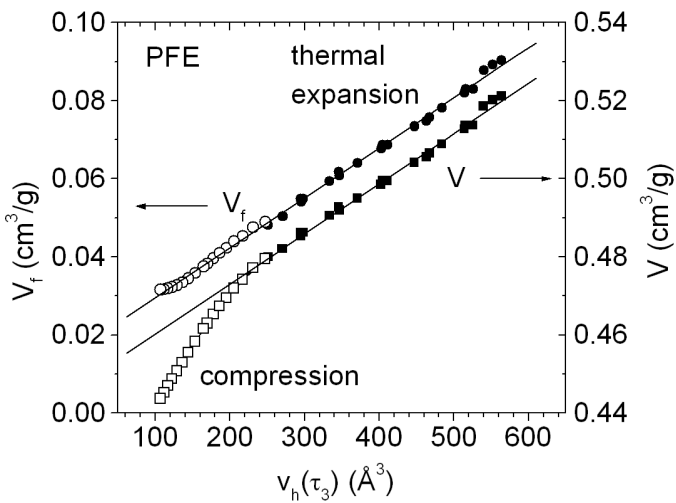

Fig. 5. Specific free volume $V_{\mathrm{f}}$ (circles) and specific total volume $V$ (squares) plotted vs. the mean hole volume calculated as $v_{\mathrm{h}}\left(\tau_{3}\right)$ for PFE. Empty symbols: compression experiments $(T=295.8 \mathrm{~K}, P=0.1-192 \mathrm{MPa})$, filled symbols: thermal expansion experiments $\left(P=10^{-5} \mathrm{~Pa}, T=T_{\mathrm{g}}+20 \mathrm{~K}-T_{\mathrm{k}}\right)$. The lines show linear fits to all data $\left(V_{\mathrm{f}}\right.$, slope $\left.=128( \pm 0.01) \times 10^{21} \mathrm{~g}^{-1}\right)$ or only to thermal expansion experiments $\left(V\right.$, slope $\left.=0.129( \pm 0.01) \times 10^{21} \mathrm{~g}^{-1}\right)$.

$199( \pm 0.01) \times 10^{21} \mathrm{~g}^{-1}$, corresponds to a volume related hole density of $N_{\mathrm{h}}=$ $N_{\mathrm{h}}^{\prime} / V=0.41( \pm 0.03) \mathrm{nm}^{-3}$.

Figure 4 shows the hole volume $v_{\mathrm{h}}$ as a function of the pressure $P$. The decrease in $v_{\mathrm{h}}$ flattens at the glass transition at $P \approx 100 \mathrm{MPa}(T=295.8 \mathrm{~K})$. In Fig. 5 plots of $V_{\mathrm{f}}$ and $V$ vs. $v_{\mathrm{h}}$, here calculated from simplicity directly from $\tau_{3}, v_{\mathrm{h}}=v_{\mathrm{h}}\left(\tau_{3}\right)$, for the thermal expansion and isothermal compression experiments, are shown. The $V_{\mathrm{f}}$ vs. $v_{\mathrm{h}}$ curve shows a linear behaviour indicating the agreements of the $N_{\mathrm{h}}^{\prime}=\mathrm{d} V_{\mathrm{f}} / \mathrm{d} v_{\mathrm{h}}$ values estimated from both types of experiments. The change in the slope of the $V$ vs. $v_{\mathrm{h}}$ curve has his origin in the compressibility of the occupied volume and disappears when taking this into account, $N_{\mathrm{h}}^{\prime}=\mathrm{d} V / \mathrm{d} v_{\mathrm{h}}-\mathrm{d} V_{\text {occ }} / \mathrm{d} v_{\mathrm{h}}$ and $\mathrm{d} V_{\text {occ }} / \mathrm{d} v_{\mathrm{h}}=\left(\mathrm{d} V_{\text {occ }} / \mathrm{d} P\right) /\left(\mathrm{d} v_{\mathrm{h}} / \mathrm{d} P\right)=$ $V_{\mathrm{occ}} \kappa_{\mathrm{occ}} / v_{\mathrm{h}} \kappa_{\mathrm{h}}$.

\section{References}

[1] L.A. Utracki, R. Simha, Macromol. Theory Simul. 10, 17 (2001).

[2] G. Dlubek, J. Pionteck, D. Kilburn, Macromol. Chem. Phys. 205, 500, 512 (2004).

[3] G. Dlubek, A. Sen Gupta, J. Pionteck, R. Krause-Rehberg, H. Kaspar, K.H. Lochhaas, Macromolecules 37, 6606 (2004).

[4] G. Dlubek, J. Wawryszczuk, J. Pionteck, T. Goworek, H. Kaspar, K.H. Lochhaas, Macromolecules, in press.

[5] J. Kansy, Nucl. Instrum. Methods Phys. Res. A 374, 235 (1996) and private communication, 2002.

[6] Principles and Application of Positron and Positronium Chemistry, Eds. Y.C. Jean, P.E. Mallon, D.M. Schrader, World Scientific, Singapore 2003. 